\title{
Analysis on the Influencing Factors of M-learning Behavior Intention of Postgraduates
}

\author{
Tang Ren Ju, Chen Min, Yang Xi Mei, Liao Jia Li \\ College of Computer and Information Science, Chongqing Normal University, Chongqing, China \\ Email address: \\ 1508063309@qq.com (Tang Ren Ju),1358516383@qq.com (Chen Min),yxm1601@126.com (Yang Xi Mei), \\ 1134970980@qq.com (Liao Jia Li)
}

\section{To cite this article:}

Tang Ren Ju, Chen Min, Yang Xi Mei, Liao Jia Li. Analysis on the Influencing Factors of M-learning Behavior Intention of Postgraduates. Science Innovation. Vol. 9, No. 3, 2021, pp. 114-117. doi: 10.11648/j.si.20210903.17

Received: April 2, 2021; Accepted: May 10, 2021; Published: May 24, 2021

\begin{abstract}
With the development and progress of science and the continuous development of new branches in the field of technology, great changes have taken place in the field of education and learning. Some new technologies provide timely information access for mobile learning, so that users can access information resources anytime and anywhere. Mobile learning has been applied and developed rapidly in college students. It is of great practical significance to study the influencing factors of M-learning behavior intention of Postgraduates for effectively improving their own development. Based on the theory of planned behavior, this study explores the factors that affect the behavior intention of M-learning of postgraduates. The purpose is to provide some meaningful suggestions and measures to help postgraduates better carry out m-learning. Based on the theory of behavior, the questionnaire was designed. In this study, random sampling method was used to conduct the survey. The subjects of the survey were graduate students of $* *$ Normal University. A total of 322 questionnaires were collected. The results show that the main influencing factors of M-learning behavior intention of postgraduates are classmate influence, usefulness cognition and self-efficacy. Therefore, it is necessary to improve the understanding of these aspects in order to effectively promote $\mathrm{m}$-learning behavior intention of postgraduates.
\end{abstract}

Keywords: Master Mobile Learning, Theory of Planned Behavior, Postgraduates

\section{硕士研究生移动学习行为意向影响因素分析}

\author{
唐仁菊, 陈敏, 杨喜梅, 廖家利
}

重庆师范大学计算机与信息科学学院, 重庆, 中国

邮箱

1508063309@qq.com（唐仁菊），1358516383@qq.com（陈敏），yxm1601@126.com（杨喜梅），1134970980@qq.com（廖家利）

摘要：随着科学的进步和发展，以及技术领域新分支的不断延伸，教育领域发生了巨大的变化。一些新技术为移 动学习提供了及时的信息访问, 让使用者能够随时随地的获取信息资源。移动学习在高校学生群体中得到了快速 的应用和发展。研究硕士研究生的移动学习行为意向影响因素,对于有效提升硕士研究生的自身发展具有重要的现 实意义。本研究基于计划行为理论去探讨影响硕士研究生移动学习行为意向的因素, 研究的目的是为了帮助硕士研 究生更好的去进行移动学习提供一些有意义的建议和措施。基于计划行为理论进行问卷的编写, 以线上发放问卷 为主要的方式进行资料的收集。本研究采用的研究方法是随机抽样的方法,本次研究的调查的对象为 $* *$ 师范大学的 硕士研究生, 问卷一共回收了 322 份。研究结果发现影响硕士研究生移动学习行为意向的因素主要有同学影响、有 用性认知、自我效能这几个方面, 因此要有针对性的提高这些方面的认识才能有效促进硕士研究生进行移动学习 的行为意向。 
关键词: 硕士研究生, 移动学习, 计划行为理论

\section{1. 引言}

在过去几年中, 具有高技术能力的移动设备的使用 有所增加, 这些设备反映了现代教育的趋势, 为学习者 提供了对所需要的信息进行即时访问的技术支持, 学习 者可以借助这一种学习方式进行移动数字资源的学习。 研究表明, 基于计划行为理论的移动学习的相关研究较 少。Shu Hsu Lin等人基于计划行为理论对台湾、中国、 印尼和越南学生对移动学习的行为意向进行研究, 提出 了一个概念模型和理论框架, 通过计划行为理论 (TPB) 结合不同的结构来考察跨文化学生对语言学习的态度的 影响[1]。Sevda Kucuk等人基于计划行为理论来构建结构 方程模型来研究医学生使用移动学习行为意向 [2]。王进 等人研究基于计划行为理论的专科护生手机移动学习行 为意向影响因素分析 [3]。通过查阅国外的相关研究,发现 计划行为理论被应用与多个领域的研究, 国内目前对移 动学习的研究多集中于移动资源建设,其次为基础理论 研究, 对于移动学习影响因素特别是以硕士研究生为研 究对象的研究还尚未见报道。移动学习是一种能够随时 随地借助互联网获取学习资源的一种学习方式, 并且具 是多种学习方式的载体, 例如智能手机、 iPad、笔记本 电脑等都可以作为它的载体。对于硕士研究生群体来说, 他们既面临着科研学习压力,另一方面又拥有大量的课 余时间, 从传统课堂上得到的知识是有限的, 想要展开进 一步的学习并取得好的科研成果, 就要求硕士研究生群 体发挥自己的主观能动性去学习。而利用移动学习这种 方式可以让硕士研究生不受时间地点的限制去获取信息 资源, 达到提升自己能力的目的。所以, 本次研究的目的在 于探讨影响硕士研究生移动学习行为意向的影响因素, 提出相应的建议来改善当前移动学习的环境, 促进硕士 研究生更好的利用移动学习这一种学习方式进行学习, 也为移动学习的推广提供一些参考。

\section{2. 对象和方法及理论基础}

\section{1. 对象和方法}

本研究采用随机抽样的方法进行调查,调查的对象为 **师范大学的硕士研究生, 调查问卷主要以线上的形式进 行发放和填写, 本次调查共发放问卷322份, 回收问卷 322 份。利用问卷星进行数据的收集, SPSS进行数据的分析。 通过查阅中国知网、百度学术、爱学术等网络平台搜索相 关文献进行文献分析, 根据文献分析进行资料收集和问卷 设计。基于计划行为理论影响硕士研究生移动学习行为意 向的因素调查问卷主要包含二个板块:第一板块为调查对 象的基本信息, 第二板块是以量表构成的硕士研究生移动 学习行为意向影响因素调查问卷, 以计划行为理论为基础 的问卷一共编制了四个大类, 十个部分和二十八个条目组 成。预实验 $\alpha$ 值为 0.975 , 说信效度良好。

\section{2. 理论基础}

计划行为理论是 Ajzen (1985) 基于理性行为理论和 多维属性态度理论提出的, 主要通过信息加工的方式分析 计划行为理论模型中的行为态度 (Behavioral Attitude)、 主观规范 (Subjective Norm) 和知觉行为控制 (Perceived Behavioral Control) 三个变量对个体行为意向的作用机制, 根据行为意向的中介作用对个体实际行为进行解释和预 测的一般决策过程的理论 [117]。计划行为理论认为人去做 一件事情的时候是受其本身行为意向影响的, 其本身的行 为意向会决定人的行为, 而人本身的行为意向会受到自身 的行为态度、主观规范及知觉行为控制这 3 个变量的影响。 这 3 个变量的相关关系即本研究的理论框架, 因此本研究 采用计划行为理论作为基本分析框架, 沿袭原有模型的框 架构建出硕士研究生移动学习使用意向的影响因素研究 模型。见图1.

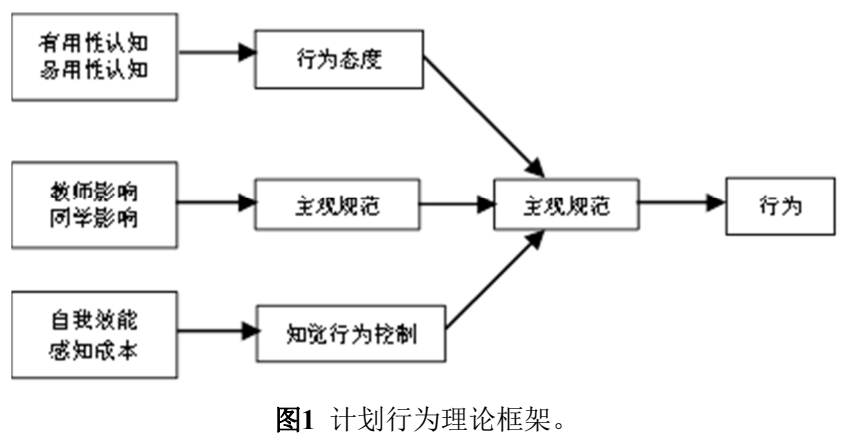

\section{3. 结果}

\section{1. 硕士研究生移动学习的影响因素得分}

表1 硕士研究生移动学习的影响因素得分。

\begin{tabular}{llll}
\hline 项目 & 最小值 & 最大值 & 均分 $(\bar{x} \pm \mathbf{S})$ \\
\hline 行为态度 & 3.00 & 15.00 & $3.81 \pm 0.05$ \\
有用性认知 & 3.00 & 15.00 & $3.90 \pm 0.05$ \\
易用性认知 & 3.00 & 15.00 & $3.87 \pm 0.05$ \\
主观规范 & 3.00 & 15.00 & $3.83 \pm 0.04$ \\
学校教师 & 2.00 & 10.00 & $3.77 \pm 0.42$ \\
同学影响 & 2.00 & 10.00 & $3.86 \pm 0.04$ \\
知觉行为控制 & 3.00 & 15.00 & $3.78 \pm 0.04$ \\
自我效能 & 3.00 & 15.00 & $3.85 \pm 0.04$ \\
感知成本 & 3.00 & 15.00 & $3.48 \pm 0.05$ \\
行为意向 & 3.00 & 15.00 & $3.74 \pm 0.04$ \\
\hline
\end{tabular}

从硕士研究生移动学习的影响因素得分表可以知道 主观规范、行为意向、行为态度和知觉行为控制的得分都 处于一个中等的水平, 从硕士研究生移动学习的影响因素 得分表可以知道有用性认知均分 $(3.90 \pm 0.05)$ 最高而感知 成本均值 $(3.48 \pm 0.05)$ 最低。 


\section{2. 相关性分析}

表2 相关分析。

\begin{tabular}{lll}
\hline 自变量 & $\mathbf{r}$ 值 & $\mathbf{P}$ \\
\hline 主观规范 & 0.573 & 0.00 \\
行为态度 & 0.639 & 0.00 \\
知觉行为控制 & 0.667 & 0.00 \\
\hline
\end{tabular}

利用SPSS软件和相关方法对硕士研究生行为意向和 主观规范、行为态度和知觉行为控制这几个影响因素进行 Pearson相关分析, 得到的结果如下表2。

根据相关分析表可知, 主观规范、行为态度和知觉行 为控制与行为意向之间的相关性 $\mathrm{r}$ 值均 $>0.5$ 且 $\mathrm{P}$ 值均 $<0.05$, 这些数值说明了说明主观规范、行为态度和知觉行为控制 与行为意向之间的相关性是非常明显的, 说明了它们之间 的相关性较高。

\section{3. 多元回归分析}

利用SPSS软件对影响硕士研究生移动学习行为意向 的 9 个相关因素进行多元线性回归分析, 其中因变量为行 为意向, 自变量为行为态度、有用性认知、易用性认知、 感知成本、知觉行为控制、同学影响、学校教师、自我 效能、主观规范。分析结果中有 6 个自变量进入方程, 分 别为知觉行为控制、行为态度、感知成本、同学影响、有 用性认知与自我效能, $\mathrm{R} 2$ 值为 0.618 , 共解释方程总变 异的 $61.8 \%$ 。详见表 3 。

表3 多元回归分析。

\begin{tabular}{lllll}
\hline 项目 & $\begin{array}{l}\text { 偏回归系 } \\
\text { 数 }\end{array}$ & $\begin{array}{l}\text { 标准化偏 } \\
\text { 回归系数 }\end{array}$ & $\mathbf{t}$ 值 & $\mathbf{P}$ \\
\hline 常量 & 0.164 & & 0.911 & 0.363 \\
知觉行为控制 & 0.263 & 0.275 & 5.028 & .000 \\
感知成本 & 0.164 & 0.197 & 4.474 & .000 \\
同学影响 & 0.180 & 0.182 & 3.686 & .000 \\
有用性认知 & 0.116 & 0.135 & 2.292 & .023 \\
自我效能 & 0.118 & 0.115 & 2.011 & .045 \\
\hline
\end{tabular}

\section{4. 讨论}

随着科学技术和互联网的高速发展,各种各样的移动 设备随处可见。线上学习资源也随着互联网时代的快速发 展变得越来越丰富,这使得学生使用移动设备进行移动学 习时不再局限于上网查找资料,而是包含学习课堂知识、 课外知识、生活技能等一系列活动。可见移动学习是一种 非常方便的学习方式。从表1的结果可以看出,硕士研究生 移动学习行为意向评分为 $(3.74 \pm 0.04)$ 分, 说明了行为意向 的得分处于中上水平, 从而知道了硕士研究生对移动学习 的行为意向表示赞同的,其中有用性认知的评分是最高的, 为 (3.90土0.05) 分, 而得分最低的变量是感知成本为 (3.48 \pm 0.05$)$ 分。说明移动学习这一种学习方式对硕士研究 生来说是一种有用的,但进行移动学习的成本也在影响着 他们的行为。随着知识付费时代的发展, 使得越来越多的 优质学习资源往往也需要花费大量的金钱。因此,推出一 些性价比较高的学习资源、物美价廉的设备资源与价格合 理的网络课程,会适当地促进硕士研究生进行移动学习。
硕士研究生也可以对网络学习资源进行全方面和多方位 地探索,在多元的信息网络环境中找到最适合自己使用的 学习资源。

\section{5. 结果与建议}

\section{1. 加强硕士研究生的自我能效感}

知觉行为控制是指促进或阻碍行为意向的一些必要 的相关因素。从表 2 中可以看出,知觉行为控制与硕士研究 生移动学习的行为意向呈正相关( $\mathrm{r}$ 值为 0.667$)$ 也就是说它 们是正向相关的,由表 3 可以看出知觉行为控制的标准化 偏回归系数的值为 0.275 , 说明对硕士研究生进行移动学习 的这个行为意向影响程度比较深的是知觉行为控制这个 自变量。从表 3 可以看出对硕士研究生移动学习行为意向 有影响的自变量有自我效能(自我效能的偏回归系数为 $0.115)$ 。自我效能主要是指硕士研究生认为自己有能力去 学习的一种自信程度。由此可知硕士研究生对移动学习过 程越有信心, 就越容易产生使用移动学习的行为意向。自 我效能感的增强能使硕士研究生进行移动学习的过程产 生必要的信心,他们相信自己可以使用移动学习,进而会进 行多次尝试, 而有了成功的移动学习案例,也会对下一次使 用移动学习增加信心, 因为有了成功的经验,会增强自信心 即"我能行"的信念从而达到促进硕士研究生利用移动设 备进行移动学习的这样的一种行为。

因此,建议硕士研究生在移动学习内容的选择上,可以 根据自己的自我认知选择一些适合自己的学习内容,由易 到难,循序渐进地学习; 在进行移动学习的时候可以尽量的 去选择自己喜欢的学习方式; 在移动学习过程中,应该具有 目的性和规划性,对所学内容进行有规划地学习。硕士研 究生利用移动学习这一种良好的学习方式, 对所学的知识 进行一种系统的、深度的学习, 进一步去拓展知识的广度, 去挖掘知识的深度, 从而促进硕士研究生对所学知识建构 系统的框架, 有利于硕士研究生的知识网络体系的形成。 转变传统学习方式的单一获取形式,利用新的学习方式进 行多元的、深度的、系统的学习, 从而加快知识的转化效 率,达到提升硕士研究生自身对移动学习信心的目的。同 时学校也可以为硕士研究生的移动学习提供便利,为学生 创造一个合适的学习环境与学习氛围,增强硕士研究生的 自我效能感,促进硕士研究生持久深度地进行移动学习。

\section{2. 营造良好的移动学习氛围}

俗话说“近朱者赤,近墨者黑”,人们很容易受到周围事 物的影响,不同的影响会成就不一样的人生。从表 2 的结果 可以知道,主观行为规范与行为意向是呈现正向相关的其 中 $\mathrm{r}$ 的值为 0.573 , 说明了硕士研究生身边重要的人(朋友、同 学、师长、家长等)对使用移动学习的这一种学习方式的 相关态度和行为会影响硕士研究生对使用移动学习的态 度。硕士研究生阶段与本科教育不同之处在于硕士研究生 要学会独立自主的进行学习, 研究生在导师的带领下掌握 基本的项目研究方法和技术去开拓本专业领域的一些知 识并取得初步成果。这个阶段的硕士研究生学习主要采用 一种以授课为辅,自学为主的学习方式, 教师往往只起到辅 
助作用, 更多的知识需要依靠学生自己探究学习与团队合 作。所以硕士研究生使用移动学习的行为会受到周围同学 的影响, 研究团队成员对移动学习的行为态度也会影响他 们。

因此,建议在校园中营造“同学带同学,教师带同学”的 移动学习氛围。同学之间可以相互交流学习方式,分享学 习相关知识和相关资源,例如学习 app, 微信公众号,网站等, 让在移动学习上获得成功的同学分享自己的学习方法, 可 以促进其他同学使用移动学习的行为意向。同学之间的竞 争关系也能潜移默化地影响到其他同学去使用移动学习。 老师在上课的时候可以试着结合移动学习来给学生上课, 带动学生去使用移动学习。相信通过老师和同学一起努力 过后, 可以营造一种适合老师和同学的移动学习氛围,从而 促进更多的硕士研究生进行移动学习, 促进硕士研究生使 用移动学习的行为意向的产生。

\section{3. 提高硕士研究生对移动学习的认知}

从研究数据中可以知道硕士研究生移动学习行为意 向受行为态度的影响并且是正向的影响, 其中相关系数为 0.639。从表格3回归分析可以知道有用性是影响行为意向 最深的一个变量。可以看出硕士研究生学习的目的性较强, 更加倾向于专注某个领域的研究,他们会选择相对新的研 究热点或者自己感兴趣的研究方向进行学习。“聚沙成 塔”“滴水穿石”, 知识是需要不断累积的, 只进行碎片化 的学习是不能够了解全部知识的, 只有积少成多对所学内 容进行深度的理解与整合,才能对研究的内容理解比较全 面、深刻。

因此,建议移动学习的资源建设者、平台开发者去调 查社会的实际情况, 深刻了解用户的需求从而去完善移动 学习的资源, 提高移动学习资源的可利用性和实用性。例 如深度调查硕士研究生对学习资源的实际需求和建议,进 而对移动学习的资源进行完善,让硕士研究生切身体会到 学习资源的有用性。发挥硕士研究生学习的自主性、独立 性、创造性与接受力强的特点, 转变硕士研究生对移动学 习的观念和态度, 最终促进其行为意向的产生和持续。

\section{6. 结语}

综上所述,从研究的数据来看计划行为理论对移动学 习行为意向影响因素具有良好的解释能力和预测能力, 用 来探究硕士研究生移动学习行为意向的影响因素是具有 一定的解释能力和可行性的。在探究硕士研究生移动学习 行为意向的影响因素中发现了知觉行为控制,感知成本,同 学影响,有用性认知,自我效能这 5 个方面影响的显著性较 为明显。说明这 5 方面是影响硕士研究生移动学习行为意 向的主要因素。可以从这 5 个方面去提升硕士研究生使用 移动学习的使用能力, 促进硕士研究生更好的去进行学习。
本研究希望能够为硕士研究生移动学习领域提供理论参 考。

\section{参考文献}

[1] Lin Shu Hsu et al. Behavioral intention towards mobile learning in Taiwan, China, Indonesia, and Vietnam[J]. Technology in Society, 2020, 63

[2] Kucuk Sevda et al. A Model for Medical Students' Behavioral Intention to Use Mobile Learning[J]. Journal of Medical Education and Curricular Development, 2020, 7 : $2382120520973222-2382120520973222$.

[3] 王进,邹晶莹,林婕.基于计划行为理论的专科护生手机移动 学习行为意向影响因素分析 [J]. 护理研 究,2017,31(30):3849-3851.

[4] 马艺颖. 计划行为理论视角下济南市居民亲子旅游行为意 向研究[D].西北师范大学,2020.

[5] 周彩霞.基于 TPB和DTPB理论的大学生移动学习影响因素 研究 [J].河西学院学报,2016,32(05):39-45.

[6] 袁柳. 大学生移动学习使用意愿的影响因素研究[D].江西 财经大学,2019.

[7] 谢聪爽. 基于UTAUT模型的大学生接受手机移动学习影响 因素研究 $[D]$.云南师范大学,2017.

[8] 丁慢慢. 大学生基于智能手机的移动学习状况研究 [D]. 南 昌大学, 2017 .

[9] 安丹丹. 大数据时代下基于随机抽样法的大学生移动学习 动机特征分析[J].无线互联科技,2020,17(09):98-100.

[10] 王金涛.大学生采纳移动学习行为影响因素研究——以高 等师范院校为例 [J].中国远程教育,2015(01):49-54.

[11] 宋宏敏. TPB视角下儿童绘本消费行为意向影响因素 分析 [D].内蒙古大学, 2020 .

[12] User Acceptance of Information Technology: Toward a Unified View[J]. MIS Quarterly,2003,27(3).

[13] Rouhollah Tavallaee and Sajjad Shokouhyar and Fatemeh Samadi. The combined theory of planned behaviour and technology acceptance model of mobile learning at Tehran universities[J]. Int. J. of Mobile Learning and Organisation, 2017, 11(2): 176-206.

[14] 纪兰.基于计划行为理论的“组织行为学”移动学习模式设计 [J].江苏商论,2018(10):139-141.

[15] 李佳卧,张秀兰.大学生移动学习特点及影响因素 [J].办公自 动化,2021,26(01):38-40+9. 